Cahiers « Mondes anciens »

\title{
Différence et codification : Alcmène dans Amphitryon de Plaute
}

\section{Pierre Letessier}

\section{(2) OpenEdition}

1 Journals

\section{Édition électronique}

URL : http://journals.openedition.org/mondesanciens/801

DOI : 10.4000/mondesanciens.801

ISSN : 2107-0199

\section{Éditeur}

UMR 8210 Anthropologie et Histoire des Mondes Antiques

\section{Référence électronique}

Pierre Letessier, «Différence et codification : Alcmène dans Amphitryon de Plaute », Cahiers « Mondes anciens » [En ligne], 3 | 2012, mis en ligne le 24 juin 2014, consulté le 21 décembre 2020. URL : http:// journals.openedition.org/mondesanciens/801 ; DOI : https://doi.org/10.4000/mondesanciens.801

Ce document a été généré automatiquement le 21 décembre 2020.

\section{(c) (i) (9)}

Les Cahiers «Mondes Anciens » sont mis à disposition selon les termes de la licence Creative Commons Attribution - Pas d'Utilisation Commerciale - Pas de Modification 4.0 International. 


\title{
Différence et codification : Alcmène dans Amphitryon de Plaute
}

\author{
Pierre Letessier
}

1 Avec son sujet mythologique et ses personnages divins, Amphitryon occupe une place à part dans la production de Plaute. Le prologue présente la pièce comme une tragicomédie (v. 59), ce qui a même amené la critique à lui chercher un modèle original grec qui ne soit pas une comédie nouvelle ${ }^{1}$, comme cela a pourtant toujours été le cas pour les comédies romaines (palliatae). Qu'une pièce si singulière comporte des personnages exceptionnels n'a rien de surprenant. C'est tout particulièrement le cas d'Alcmène. Charles GUITTARD (1998, p. 48) écrit ainsi qu' "Alcmène, par son amour plein de dignité, est complètement différente de l'image caricaturale des épouses et des mères que l'on trouve dans les autres comédies $»^{2}$. Amphitryon lui-même la désigne comme «la meilleure épouse de Thèbes ", ce qui incontestablement fait d'elle un personnage féminin à part: quand les épouses de la palliata se caractérisent par leur méchanceté (malitia), Alcmène, elle, est une uxor optima (v. 676-677).

2 Si la différence d'Alcmène est indéniable, se pose néanmoins la question de son interprétation. Celle-ci dépend du statut générique que l'on prête à Amphitryon et, plus largement, de la conception que l'on se fait du théâtre de Plaute, selon que l'on considère qu'il se caractérise par une variété et une fantaisie qui exclut finalement toute codification $^{3}$ ou au contraire, comme nous le croyons, par un code connu et attendu du public, celui de la palliata (LETESSIER 2010). Si l'on prête à la pièce de Plaute une dimension autre qu'une simple comédie, on aura certes tendance à exclure le personnage féminin de la sphère comique. Abordée sous un angle sérieux, la différence d'Alcmène a ainsi été pensée comme un écart qui lui ferait dépasser son simple statut d'épouse de comédie pour devenir l'idéal de la matrone romaine ${ }^{4}$ ou une héroïne tragique ${ }^{5}$. Une telle approche est basée sur la conception d'un théâtre marqué par la variété. Mais si on considère qu' Amphitryon reste une palliata (ce que l'hypothèse d'une source extérieure n'interdit pas), entendue comme spectacle codifié, il faut alors chercher à comprendre la différence d'Alcmène en la replaçant au sein du code comique. Deux études assez récentes, celles de PHILLIPS (1985) et de CHRISTENSON (2001), ont ouvert la voie à une telle réinterprétation en 
dégageant, à l'encontre de la tradition, une dimension grotesque du personnage. Mais elles l'ont fait en maintenant encore la perspective tragique et sans réinscrire systématiquement ce rôle dans la codification de la palliata. C'est précisément ce que nous voudrions faire ici : sans passer par la question du rapport entre la tragédie et la comédie ${ }^{6}$ , réinscrire le personnage d'Alcmène dans le code des rôles de la palliata pour comprendre ce que recouvre sa différence.

3 Alcmène porte un masque d'épouse de comédie ; or, le masque d'un personnage n'est pas seulement associé à un type de costume et de perruque, il implique aussi un certain nombre de caractéristiques narratives et spectaculaires qui créent un horizon d'attente chez le public. Si l'on se place du point de vue de la réception spectaculaire du théâtre de Plaute, la «différence » d'un personnage doit donc d'abord se comprendre par rapport à l'attente d'énonciation gestuelle et verbale que suscite son masque, c'est-à-dire son rôle sa persona $a^{7}$. Or, nous avons montré ailleurs que la codification de la palliata était constituée par un ensemble non de règles strictes mais de variations (LETESSIER 2010), ce qui permet de concilier l'impression de variété que peut donner le théâtre de Plaute avec l'existence d'un code théâtral. Ce qui a été si souvent pointé comme différence dans le personnage d'Alcmène relèverait-il en fait de la variation? Dans ce cas, la « différence » d'Alcmène, uxor optima, ne constitue pas un écart ou une rupture avec le code comique, qui tire le personnage du côté d'un idéal romain ou de la tragédie, mais une façon traditionnelle de faire évoluer un rôle dans une comédie romaine.

\section{La codification traditionnelle de l'uxor de palliata}

4 Afin de pouvoir percevoir la différence d'Alcmène, commençons par dresser la codification de l'épouse de palliata. Bien que peu représenté, ce qui se traduit par un flottement de la dénomination dans les didascalies ${ }^{8}$, ce rôle apparaît néanmoins dans quelques comédies ${ }^{9}$ et fait l'objet, dans plusieurs autres, de nombreux discours ${ }^{10}$. Ce qui nous permet de dégager des éléments communs spécifiques, sans prendre en compte encore le cas d'Alcmène dans Amphitryon.

5 Pour une part, la codification des rôles (personae) est narrative ${ }^{11}$ : à chaque masque est associé un ensemble de caractéristiques morales et sociales qui déterminent un certain type de relations avec les autres rôles et, par-là, sa place dans l'histoire. L'épouse se définit essentiellement par son lien conjugal avec son mari, c'est-à-dire avec un senex ${ }^{12}$. Cette relation confine à la dépendance. S'il y a une uxor sur scène, il y a aussi un senex, alors que l'inverse n'est pas vrai. Elle ne se définit donc pas par le fait d'avoir un enfant et d'être mater ${ }^{13}$ - ce qu'elle est toujours pourtant ${ }^{14}-$, au contraire du senex qui, lui, est avant tout un pater.

6 L'épouse de comédie est non seulement inséparable de son mari, mais elle est crainte par celui-ci. Elle est, en effet, dotée (uxor dotata). Cette dot est une source de malheurs domestiques pour le mari ${ }^{15}$ : elle la rend dépensière ${ }^{16}$ et lui donne le pouvoir - imperium ${ }^{17}$ - sur son époux. Si une femme sans dot est soumise à son mari, une femme dotée veut le soumettre. Elle est ferox - terme qu'on applique aux chevaux indomptés (Menaechmi, v. 766-767 et 863). Et elle est d'autant plus crainte par son mari que celui-ci est amoureux - senex amator. Dans les comédies où apparaît l'épouse, en effet, le senex, qui se définit par son opposition aux amours de son fils, est toujours lui-même amoureux d'une femme une esclave ou une prostituée ${ }^{18}$. Aussi toute rencontre entraîne-t-elle un conflit et une scène de ménage. L'épouse a pour fonction d'empêcher son époux de se comporter 
comme un adulescens, c'est-à-dire de lui retirer une caractéristique qu'il emprunte à un autre rôle, et de le ramener à un comportement conforme à son rôle de senex, qui est étranger à l'amour. En ce sens, elle est la garante de la codification. C'est pourquoi il ne peut y avoir de bonne épouse (bona uxor) sur la scène comique ${ }^{19}$. L'épouse est « mauvaise " pour son mari et se trouve qualifiée d'adjectifs négatifs : mala, odiosa, improba, incommoda, etc. C'est pourquoi aussi les senes mariés souhaitent souvent la mort de leur femme ${ }^{20}$. Pour un vieux de comédie, une bonne épouse est une épouse morte ${ }^{21}$. Ainsi, la place de l'épouse dans le schéma actantiel de la comédie se définit également par sa relation avec le senex. C'est seulement dans la mesure où elle s'oppose au senex, qui est un des opposants traditionnels du jeune homme, qu'elle se retrouve, de façon indirecte donc, adjuvante de l'adulescens ${ }^{22}$.

La codification de l'épouse est aussi spectaculaire. Elle consiste en un masque (wILES 1991, p. 132-133), une perruque (avec des tresses et des rubans) et un costume (la palla avec une couleur spécifique) caractéristiques et elle implique aussi une dynamique et une gestuelle propres, c'est-à-dire une façon de mettre en mouvement ce masque et ce costume. La codification du rôle s'étend enfin jusqu'à la métrique et au mode d'interprétation qu'elle suppose. Presque les trois quarts des scènes comportant une épouse sont écrites en canticum, essentiellement en canticum monomètre. L'épouse de comédie danse ${ }^{23}$. C'est un rôle peu représenté mais remarqué, qui fait l'objet d'une exploitation spectaculaire forte. Et son partenaire privilégié est le senex. Or, le canticum monomètre marque les rencontres importantes et conflictuelles des comédies (LETESSIER 2007). Le conflit entre les époux et l'imperium de l'uxor prennent ainsi une forme "chorégraphique"; de même qu'elle contrecarre les projets amoureux de son mari, l'épouse essaie d'avoir la maîtrise de l'espace et des mouvements de son mari. Les deux scènes de final d'Asinaria et de Casina sont à ce titre emblématiques : parce qu'elle l'a surpris avec une fille, l'épouse y impose à son mari un mouvement de sortie de scène. Au sens propre, l'épouse mène la danse et malmène son partenaire. Le vocabulaire désignant ces scènes de rencontre est celui de la guerre et des coups ${ }^{24}$ et le vieux qui affronte sa femme ne cesse de répéter qu'il est mort ( perii, occidi ${ }^{25}$ ) - c'est-à-dire que son rôle de senex amator est terminé. Aussi la rencontre entre les époux s'accompagne-t-elle également d'un certain type de paroles. L'épouse, qui se caractérise également par un débit verbal soutenu, multiplie les reproches et les accusations à l'encontre de son mari, avec colère - ce qui présage là encore d'une utilisation remarquable du corps ${ }^{26}$.

8 Tout ceci constitue donc l'horizon d'attente du public romain. Une épouse de comédie est une uxor mala qui danse avec son mari. Elle est l'opposée du rôle féminin le plus représenté, la prostituée (meretrix). Ainsi, sur la scène comique, l'épouse ne se définit pas par rapport à la uirgo ou à la vieille femme - anus - mais par rapport à la prostituée. L'une séduit, l'autre repousse; l'une est couverte de parfums délicieux, l'autre a une haleine puante (Asinaria, v. 893-895 et 928); l'une a la parole charmeuse, l'autre guerrière; la proximité physique est désirée avec l'une, redoutée avec l'autre ; l'une exécute une danse érotique de séduction, l'autre de combat. À l'inverse du théâtre d'Aristophane, une guerre du sexe se traduirait chez Plaute non par une abstinence mais par une pratique sexuelle. Dans Asinaria (v. 896-897), Artemona envisage de couvrir son mari de baisers pour le punir. C'est pourquoi les épouses de comédie ne sont pas adultères. Il ne s'agit pas, comme George E. DUCKWORTH (1952) l'écrit, d'un défaut épargné au «portrait » déjà bien chargé des uxores, mais d'une conséquence de la codification de l'épouse, comprise comme l'autre de la prostituée. 


\section{La situation initiale d'Amphitryon : une situation inédite et conforme à la tradition de la palliata}

9 Replacée dans le cadre du code de sa persona, la simple désignation d'Alcmène comme uxor optima la fait clairement apparaître comme un personnage d'épouse à part. Pourtant, rien n'autorise à concevoir cette différence comme une rupture avec le code. Le phénomène même de la différence est, en effet, traditionnel de l'écriture d'une palliata. Toute comédie doit contenir une part d'inédit : nouum - ce terme désignant précisément ce qui est différent de ce qu'on attend. Ainsi, les prologues disent, dans un même mouvement, la conformité de la pièce avec la codification de la palliata et la nouveauté ( LETESSIER, 2011). Il n'y a là rien de contradictoire; il s'agit au contraire d'un fonctionnement propre à un théâtre rituel. Comme tout acte religieux, la représentation d'une palliata doit se dérouler toujours de la même façon et, en même temps, elle doit être différente sous peine de provoquer l'ennui - ce qui est catastrophique dans la mesure où les dieux et les hommes sont censés prendre ensemble du plaisir au spectacle scénique ${ }^{27}$.

Le dieu Mercure, dans le prologue d'Amphitryon, présente clairement la nouveauté de la pièce :

\section{MERCURE}

Veterem atque antiquam rem nouam ad uos proferam.

Propterea ornatus in nouum incessi modum (v. 118-119).

Je vais vous apporter une vieille et ancienne histoire dans une nouvelle version.

C'est pourquoi je viens habillé d'une nouvelle façon.

11 Le costume n'est nouveau que pour Mercure. Il ne l'est pas pour le public puisque le dieu a l'apparence traditionnelle d'un esclave de comédie. La nouveauté ne vient donc pas du code spectaculaire mais de l'histoire qui va mettre en place une situation exceptionnelle pour une palliata. Ainsi, quand il énonce le sujet de la comédie (argumentum), Mercure ne cesse de donner aux spectateurs des renseignements inouïs. Première information: Amphitryon est à la guerre, il a laissé chez lui sa femme enceinte (v. 97-103). Mais plus encore que ce premier élément ${ }^{28}$, c'est le deuxième qui est surprenant pour les spectateurs : le gros ventre d'Alcmène constitue une variation majeure avec le code de l' uxor dans la palliata. Un tel costume et la démarche qui va avec sont du « jamais vu » sur la scène comique. Seules sont enceintes les uirgines qui ont été violées par un jeune homme, mais le public ne les voit jamais, elles restent hors scène. L'épouse de comédie, elle, n'est jamais enceinte : si elle est mariée à un jeune homme, elle n'a pas d'enfant et si elle est mariée à un senex, comme Alcmène ${ }^{29}$, elle a déjà des enfants en âge de se marier. Montrer une épouse avec un gros ventre, c'est donc montrer ce qui n'arrive jamais à ce rôle de l' uxor et surtout ce qui n'est jamais montré sur scène ${ }^{30}$. Deuxième information : Jupiter est couché dans la maison avec Alcmène, sous les traits de son époux Amphitryon; il l'aime et prend du plaisir avec elle (v. 104-105). Cette situation initiale de la comédie multiplie encore les variations majeures du rôle de l'épouse. D'une part, Alcmène est une épouse adultère - ce qui constitue une caractéristique contraire au code de son masque et un cas unique dans la palliata (DUCKWORTH 1952, p. 150). D'autre part, cet adultère n'en est pas tout-à-fait un, puisqu'elle croit se trouver dans les bras de son mari. Or, selon la codification traditionnelle, l'épouse ne suscite ni désir ni amour chez son époux mais haine et dégoût, ce qui rend la situation encore plus surprenante pour le public. La différence essentielle d'Alcmène, celle qui est donnée par le prologue, est donc constituée par l'amour que lui porte son mari ${ }^{31}$. 
12 La situation de la comédie, telle qu'elle est posée par le prologue, est ainsi, sur bien des points, inédite et peut sembler étrangère à la codification de la palliata, non pas tant d'ailleurs parce que certains rôles sont tenus par des dieux mais parce que les rôles de l'épouse et de son mari sont soumis à une variation majeure de leur persona. Pourtant, là encore, ce début de pièce est conforme à la tradition de la palliata. Beaucoup de comédies commencent, en effet, en présentant une situation limite qui fait croire que la pièce ne pourra pas se dérouler comme les autres, voire même ne pourra pas se dérouler du tout ${ }^{32}$. Ainsi le plaisir du spectateur de palliata devait consister en partie à voir comment le poète, en partant d'une situation qui semble contraire à la codification attendue, va finalement réussir à faire jouer une comédie qui ressemblera aux autres et comment il y parviendra, précisément en exploitant cette " différence » qui est présentée au début de la représentation. Annoncée dès le prologue, la «différence » d'Alcmène devait donc susciter un suspense traditionnel. Parce qu'elle est dépourvue du sujet de l'action le plus attendu - le jeune homme -, la comédie tourne autour du couple formé par Amphitryon et Alcmène. Or, comment écrire une palliata avec un mari qui aime son épouse et entretient avec elle une relation de concordia? Le partenaire de l'uxor est bien un senex amator comme dans les autres comédies où l'on retrouve le couple conjugal, mais cet amour est dirigé vers sa propre femme. Comment construire une action scénique sans le moteur dramatique que constitue l'opposition virulente d'une épouse « mauvaise » à son mari ? La réponse est donnée dans le prologue : en introduisant l'adultère de la femme ; mais puisque cela constitue une situation totalement inédite, une telle information ne renseigne pas le spectateur, elle ne peut avoir pour effet que de produire encore du suspense.

\section{Différence et inédit}

Dans un premier temps, Plaute exploite pleinement la différence de ce rôle et de cette relation de concordia entre les époux pour offrir au public un spectacle inédit. Alcmène apparaît pour la première fois en compagnie de Jupiter déguisé en Amphitryon, c'est-àdire en senex de comédie. Or, leur scène, qui survient tard dans l'économie de la pièce (Plaute faisant durer l'attente des deux rôles qui sont présentés, dès le prologue, comme exceptionnels) suit d'abord un schéma conventionnel en ce qui concerne Alcmène. Elle retient, en effet, son mari et l'empêche de s'éloigner de la porte de la maison - c'est-à-dire essaie de maîtriser ses mouvements -, l'interroge sur les motivations de son départ (v. 502-503) et formule une plainte traditionnelle sur le peu de considération des hommes à l'égard de leur femme (v. 508). Les premières répliques d'Alcmène sont donc conformes à son masque d'uxor ${ }^{33}$. Elles sont d'ailleurs prononcées en canticum. La même scène se retrouve dans Menaechmi, si ce n'est que l'épouse reste hors scène. Le mari y reproche précisément à sa femme de l'interroger sur ce qui motive son départ (v. 115).

14 Ce cadre conventionnel rend plus sensible le décalage des paroles du mari avec la codification attendue pour une scène entre un senex et son uxor: Jupiter, en effet, adresse à Alcmène des paroles bienveillantes. Autrement dit, si les tirades de celle-ci sont traditionnelles, leur motivation est tout-à-fait exceptionnelle: Alcmène ne veut pas laisser partir son mari parce qu'il l'aime.

JUPITER

Satin habes, si feminarum nulla est quam aeque diligam?

Cela ne te suffit pas de savoir que je t'aime comme aucune femme? (v. 509) 
Cet amour de Jupiter est comique parce qu'il s'inscrit dans une scène constitutive de la palliata : celle où un personnage est victime d'une «ludification ». Ce terme se rattache à un ensemble de mots et d'expressions construits sur ludus qui désignent tous le fait de tromper quelqu'un, de se jouer de lui sans qu'il le sache (ludere, eludere, ludificare, ludos facere). Toute comédie comporte, en effet, au moins une scène dans laquelle un personnage se joue d'un autre en le plongeant dans une fausse situation. Dans la plupart des pièces, il s'agit d'une ruse (ludus) mais la "ludification" peut prendre d'autres formes, comme le quiproquo ${ }^{34}$. Ici, Jupiter se joue d'Alcmène en lui faisant croire qu'il est son mari, Amphitryon. Mercure commente ainsi la scène en recourant à un vocabulaire explicite de la ruse : il désigne son père comme sycophanta (v.506), c'est-à-dire par un terme qui s'applique à celui qui réalise la ruse. Autrement dit, la scène qui se déroule entre le mari et la femme est "fausse » parce qu'il y a travestissement et elle se traduit, comme souvent chez Plaute, par un spectacle qui offre des variations majeures avec la codification attendue ${ }^{35}$ puisque le senex joue un mari amoureux de sa femme. Ainsi, le spectacle offert est bien celui, inédit, d'un couple qui s'aime, mais il se fait sous le mode explicite du jeu. Alcmène est une uxor optima, mais dans une scène qui se dénonce comme "fausse ». Sa différence est intégrée à la comédie par le biais du ludus, car Alcmène, victime du quiproquo, va effectivement faire éclater sa différence, c'est-à-dire donner à voir et à entendre la variation majeure qui la caractérise. Après avoir commencé la scène comme une uxor traditionnelle, elle va jouer à son tour la partition de la concordia, c'est-àdire parler et se comporter contrairement au code de son masque :

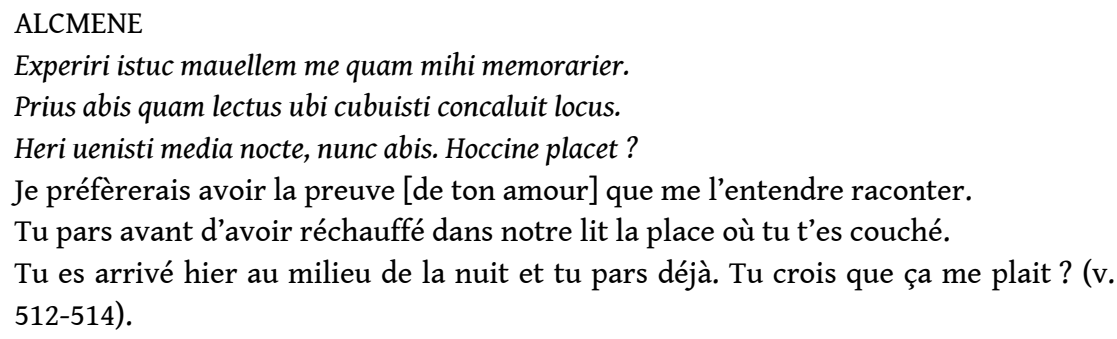

Ces paroles sont en contradiction absolue avec le masque de l'uxor. La chaleur du lit partagé avec son amant et la place devenue froide quand la femme est délaissée sont des lieux communs de la poésie érotique ${ }^{36}$. Alcmène parle ici comme une meretrix ${ }^{37}$. La réplique de Jupiter, que nous avons citée, est d'ailleurs typique de celles qui sont adressées à une prostituée : les déclarations d'amour ne s'adressent jamais à une uxor mais toujours à une meretrix. Le temps de la scène correspond également à l'exceptionnel de la situation, puisqu'il fait nuit. Là encore, le temps n'est pas celui de l'épouse mais de la prostituée $^{38}$. Ainsi les larmes que verse Alcmène en voyant partir son époux la rattachent encore à ce dernier rôle. Son mari, en effet, lui demande de ne pas abimer ses yeux exactement comme le fait le vieux Lysimaque dans Mercator lorsque la prostituée Pasicompsa pleure elle aussi ${ }^{39}$. Il s'agit de ne pas porter atteinte à sa beauté - ce qui est extraordinaire pour un masque d'épouse censé avoir une haleine fétide.

Ainsi, la concordia qui règne entre les époux se manifeste dans une scène de «ludification» et donne lieu à un spectacle inédit; la différence d'Alcmène avec la codification traditionnelle de l'épouse est telle qu'elle l'amène à jouer comme le rôle qui lui est traditionnellement opposé. L'uxor se définissant en opposition à la meretrix, toute différence marquée chez une épouse se traduit par une ressemblance avec la prostituée. On retrouve de tels jeux de ressemblance entre ces deux rôles opposés dans d'autres comédies. Ainsi, dans Mercator, Dorippa présente une autre variation majeure avec la 
codification de l'uxor: elle est silencieuse durant toute la durée d'une longue scène en diuerbium avec un cuisinier. Dans une scène qui n'est pas dansée, ce mutisme, contraire aux conventions de l'épouse bavarde, ne peut pas faire d'elle une épouse, si bien que le cuisinier la prend pour une prostituée ${ }^{40}$. Ce n'est pas un hasard non plus si, dans la grande scène de ruse finale du Miles Gloriosus (v. 1216 sq.), c'est une prostituée qui est chargée de jouer une épouse amoureuse.

\section{Différence et tradition}

Dans un deuxième temps, après avoir exploité la scène de concordia et présenté la différence d'Alcmène, c'est-à-dire après avoir fait jouer la variation d'une uxor aimée et aimante pour donner une scène inédite, Plaute va construire toute l'action sur le quiproquo : en étendant la « ludification » à tous les personnages mortels, il va mettre fin à cette concordia et permettre au public de retrouver des scènes traditionnelles entre époux. Avec un plaisir d'autant plus grand qu'elles opposeront des personnages qui se caractérisent précisément dans la pièce par leur absence de relation conflictuelle.

La rencontre entre Alcmène et le véritable Amphitryon est précédée par de nombreux apartés dans lesquels le mari dit l'amour qui l'unit à sa femme et celle-ci, la joie qu'elle a de le revoir si vite. Autrement dit, les deux masques commencent pas dire leur différence avec le code traditionnel de la palliata. En l'interpelant comme la meilleure des épouses, uxor optima (v. 676-677), en la saluant avec un plaisir affiché à deux reprises (v. 676, laetus ; v. 681, gaudeo) et en s'enquérant de sa santé, le mari se comporte à l'encontre de la codification attendue selon laquelle l'époux ne peut pas être heureux de voir sa femme et ne souhaite que sa mort. Mais, par le jeu du quiproquo, les bonnes paroles de celui-ci sont comprises de travers par sa femme :

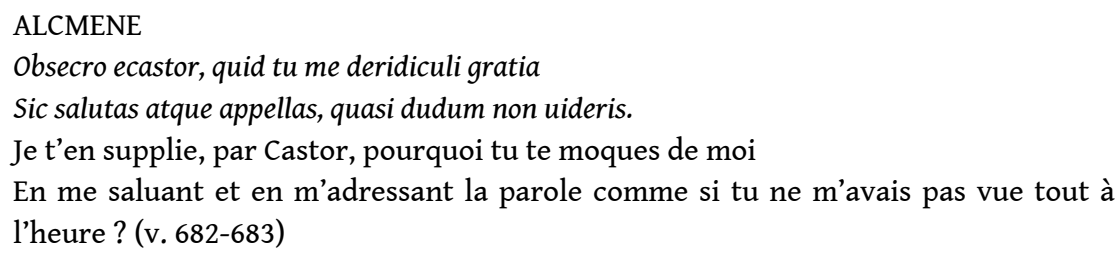

Alcmène croit que son mari se joue d'elle, qu'elle est victime d'une ludification. Aussi ne rend-elle pas à Amphitryon son salut. Ce refus d'engager une salutation ${ }^{41}$ annule les propos bienveillants du mari, casse toute réciprocité entre eux et crée les conditions d'un affrontement traditionnel. Sosie qui assiste au face à face compare l'accueil fait à son maître à celui fait à un chien - c'est-à-dire à un animal qui est aussi associé à l'épouse traditionnelle, qui suit partout son mari et peut mordre (Casina, v. 320). Ne pas saluer, c'est engager les hostilités. C'est pourquoi Amphitryon lui reprochera à plusieurs reprises de ne pas avoir répondu à sa salutation. Ecrite en canticum, la scène entre Amphitryon et Alcmène se présente donc comme une scène traditionnelle entre époux ${ }^{42}$. Alcmène $y$ développe un sentiment typique de l'uxor: la colère. Elle l'exprime même de telle façon que ses paroles et son comportement font croire à Amphitryon et à Sosie qu'elle est atteinte de folie (v. $696 ; 709 ; 727-730 ; 798)$. La scène en canticum donne donc lieu à un engagement corporel particulièrement développé qui reprend sans doute des éléments chorégraphiques caractéristiques d'une danse d'uxor mala. Ainsi, Sosie évoque la menace des coups portés par Alcmène et la compare à des frelons (v. 703-705 et 707) avant d'ajouter qu'elle est enceinte de la folie (v. 719). Cette image de la grossesse traduit le renversement du statut d'Alcmène : le ventre qui matérialisait sa différence avec le rôle 
traditionnel de l'épouse est maintenant associé à un sentiment traditionnel du rôle, la colère.

Il en sera de même pour le début de la scène qui l'opposera de nouveau à Jupiter. Elle refuse le face-à-face avec celui qu'elle prend pour son mari, le traite d'ennemi (inimicus) et lui interdit tout contact physique (v. 897-903). Autrement dit, elle se comporte alors comme une uxor traditionnelle de palliata.

Si on la considère au sein du code de la palliata, la " différence » d'Alcmène - l'amour que lui porte son mari et qu'elle partage - ne l'affranchit donc pas du rôle traditionnel de l'épouse mais lui permet au contraire de s'y inscrire pleinement. En effet, même si elle est contraire aux caractéristiques conventionnelles d'une uxor sur la scène comique, cette différence ne traduit pas un écart avec la codification attendue, mais une variation. Ainsi, la différence avec le code participe à sa validation puisqu'elle le fait jouer de l'intérieur et qu'elle constitue son mode même de fonctionnement. Pour fonctionner, en effet, le code comique ne peut pas se répéter à l'identique - ce qui provoquerait un ennui effroyable mais doit se renouveler sans cesse ou, pour ainsi dire, se répéter sous le mode de la variation.

L'exemple d'Alcmène est particulièrement intéressant dans la mesure où il montre les deux façons dont une variation majeure peut renouveler la codification attendue en produisant des éléments nouveaux et aussi des éléments traditionnels redynamisés par ces derniers : la concordia qui unit les époux produit, d'une part, de l'inédit en donnant à l'épouse des caractéristiques traditionnelles du rôle qui lui est conventionnellement opposé (celui de la prostituée) et, d'autre part, un spectacle qui est à la fois traditionnel (un conflit) et nouveau puisque ce qui motive ce conflit, par l'effet d'un quiproquo, n'est pas l'opposition habituelle d'une uxor à son mari, mais leur amour. Parce qu'elle est une uxor différente, Alcmène parle successivement comme une meretrix, puis comme une uxor traditionnelle.

Cette différence d'Alcmène avec le code de la persona de l'uxor est donc à la fois radicale, en ce qu'elle est la seule épouse aimante du théâtre de Plaute et conventionnelle, dans la mesure où toute comédie comporte nécessairement des variations radicales. Elle s'inscrit, en effet, dans un mouvement typique de la palliata où le poète joue à poser une situation et des rôles qui, par leurs variations, laissent croire au public que la pièce ne pourra pas se dérouler conformément au code attendu. On peut observer le même phénomène non seulement dans les autres comédies de Plaute mais aussi dans celles de l'autre auteur comique dont nous avons conservé des textes, Térence, qui multiplie dans ses pièces les variations majeures; lui aussi, présente d'ailleurs dans Hecyra une épouse "bonne », Sostrata, à qui on a souvent comparé Alcmène (DUCKWORTH 1952, p. 150). Une telle comparaison est pertinente, même si elle prend ici un autre sens que celui qu'on lui prête habituellement, puisque le personnage féminin ne s'affranchit pas du code comique mais le fait fonctionner précisément par sa différence (DUPONT et LETESSIER 2012, p. 180-183).

L'étude de la différence d'Alcmène nous amène finalement à revenir sur celle qui fonde la comédie même d'Amphitryon. Ce qui donne à cette pièce une place à part dans la production de Plaute (la présence active des dieux et le sujet mythologique) ne la coupe pas du code de la palliata mais la rattache à celui-ci. Toute comédie, en effet, se doit de comporter une part de nouveauté pour faire fonctionner le code. Ainsi, en un sens, les dieux ne bouleversent pas l'ordre comique mais ils le rétablissent. Grâce au quiproquo qu'il introduit, en effet, Jupiter accomplit le tour de force de faire danser (c'est-à-dire 
jouer dans un canticum) une scène de ménage à un couple qui s'aime. Il parvient donc à faire jouer une comédie en mettant fin, le temps de la représentation ${ }^{43}$, à cette relation absolument inédite et absurde qui caractérise l'histoire d'Amphitryon : l'amour conjugal.

\section{BIBLIOGRAPHIE}

CHRISTENSON D. M. (2000), Plautus : Amphitruo, Cambridge.

CHRISTENSON D. M. (2001), « Grotesque Realism in Plautus' Amphitruo », Classical Journal 96, p. 243-60.

DUCKWORTH G.E. (1952), The Nature of Roman Comedy, $1^{\mathrm{e}}$ éd., Princeton.

DUMONT J.-C. (1990), «L'imperium du paterfamilias », dans Andreau J. et Bruhns H. éd., Parenté et stratégies familiales dans l'Antiquité romaine, École Française de Rome, 129, p. 475-495.

DUPONT F. et LETESSIER P. (2012), Le Théâtre romain, Paris.

DUTSCH D.M. (2008), Feminine Discourse in Roman Comedy. On Echoes and Voices, Oxford.

FAURE-RIBREAU M. (2009), « Les personnages de l'Amphitryon de Plaute : des personnages tragicomiques? ", dans Lefebvre L. et Glinatsis R. (dir.), ACTES : Journées d'étude « Melpomène dans tous ses états. Le tragique hors de la tragédie dans la littérature latine ", Lille 3, 20 et 21 juin 2008, Mosaïque [En ligne], 1. URL : http://revuemosaique.net/wp-content/uploads/2010/03/mosaique-1-8_faureribreau.pdf

GRATWICK A.S. (1982), « Drama », dans Kenney E.J. and Clausens W.V. ed., The Cambridge History of Classical Literature, vol 2, Cambridge, p. 77-137.

GUITTARD C. (1998), Plaute: Amphitryon, Paris.

HUNTER R.L. (1985), The New Comedy of Greece and Rome, Cambridge.

LETESSIER P. (2000), « La salutatio chez Plaute, adaptation ludique d'un rituel social », Lalies 20, Paris, p. 151-163.

LETESSIER P. (2007), « Des didascalies pour les spectateurs : nature et fonctions des didascalies internes dans les comédies de Plaute », dans Calas F., Elouri R., Hamzaoui S. et Salaaoui T. éd., Le texte didascalique à l'épreuve de la lecture et de la représentation, Pessac, p. 115-130.

LETESSIER P. (2010), «Codification et variation : l'exemple du silence musical dans le théâtre de Plaute », dans Calame C., Dupont F., Lortat-Jacob B. et Manca M. éd., La Voix actée, Paris, p. 109-124.

LETESSIER P. (2011), « Du nouveau chez Plaute? des jeux de réécriture dans les comédies de Plaute », Dialogues d'Histoire Ancienne 37, 2.

MICHAUT G. (1920), Plaute, Paris.

MOORE T. J. (1995), «Tragicomedy as a Running Joke : Plautus' Amphitruo in Performance », dans Goetsch S. (ed.), How is it Played ? Genre, Performance and Meaning, Didaskalia [En ligne], Supplement 
1, vol. 1-6, consulté le 20 mars 2012. URL : http://www.didaskalia.net/issues/supplement1/ moore.html

MOORE T. J. (1998), The Theater of Plautus. Speaking to the Audience, Austin.

PACKMAN Z. M. (1999), « Feminine role designations in Plautus », AJPh 120, n² 2, p. 245-258.

PHILLIPS, J. E. (1985), « Alcumena in the Amphitruo of Plautus. A Pregnant Lady Joke », Classical

Journal 80, p. 121-126.

ROBERT J.-N. (1997), Éros romain, Paris.

SCHEID J. (2005), Quand faire, c'est croire. Les rites sacrificiels des Romains, Paris.

WILES D. (1991), The Masks of Menander. Sign and meaning in Greek and Roman Performance, Cambridge.

\section{NOTES}

1. Ainsi, on a cherché à trouver d'autres sources que la comédie nouvelle : la comédie moyenne, la farce d'Italie du Sud et même la tragédie. Sur ces différentes hypothèses, voir MOORE 1998, p. 108-109 et CHRISTENSON 2000, p. 50-55.

2. Sur la présentation d'Alcmène comme personnage exceptionnel, voir par exemple DUCKWORTH 1952 , p. 150 : «the noblest woman character in Plautine comedy » ou ROBERT 1997, p. 47 : «Plaute (...) se garde bien de peindre l'amour conjugal. Sa seule exception concerne le sentiment réel et exemplaire d'Alcmène pour Amphitryon ».

3. Considérer qu'il y a un code mais qu'une comédie est une suite de ruptures avec ce code, annule finalement l'existence même de celui-ci.

4. Pour G Michaut (1920, p. 92), Alcmène est «l'épouse vraiment digne de ce nom ». DUCKWORTH (1952, p. 257) la présente comme le personnage féminin le plus proche de la vie réelle. Voir aussi HUNTER 1985, p. 126 et GUITTARD 1998, p. 48-49 : « le personnage d'Alcmène se présente [...] comme un véritable éloge de la matrone romaine »; elle « donne déjà, au début du II ${ }^{\mathrm{e}}$ siècle, l'image de la parfaite matrone telle qu'elle sera célébrée dans les éloges funèbres et les inscriptions funéraires ».

5. Voir ERNOUT, dans la notice de l'Amphitryon $(1989$, p. 6) : «Un personnage conserve à travers toute la pièce une dignité et un sérieux qui ne se démentent pas : c'est Alcmène. [...] elle sait trouver pour se défendre des accents d'une noblesse simple et touchante qui ne détonneraient pas dans une tragédie. » Voir aussi GRATWICK (1982, p. 109-110 et 130). Une troisième voie d'étude est celle des gender studies. Voir, par exemple, D.M. DUTSCH (2008) qui distingue deux voix d'Alcmène, une masculine et une féminine.

6. Sur cette question, voir notamment T.J. MOORE (1998, chapitre 6) qui étudie la pièce comme un combat entre les genres où la comédie l'emporte sur la tragédie et M. FAURE-RIBREAU (2009) qui étudie la dimension tragique comme élément ludique, en particulier dans le premier monologue d'Alcmène.

7. Le même terme désigne le masque et le rôle : persona.

8. PACKMAN 1999 : on trouve les termes de matrona, uxor et mulier.

9. Amphitryon, Asinaria, Casina, Mercator et aussi Menaechmi et Stichus.

10. Les comédies sont pleines de paroles qui donnent les caractéristiques des rôles. Ces paroles prononcées sur les épouses constituent des sources de plaisanteries mais elles activent aussi la codification du rôle.

11. Sur la codification des rôles dans la palliata, voir DUPONT et LETESSIER 2012, p. 108-118. 
12. On trouve des épouses mariées à un adulescens dans deux comédies : Stichus et Menaechmi. Mais ce couple adulescens-uxor constitue à chaque fois une combinaison contraire au code, une variation extraordinaire, dans la mesure où l'adulescens se caractérise par la recherche du plaisir et du sexe, et où par définition, il n'est pas marié. Ainsi à la fin de Menaechmi, le jeune homme vend tous ses biens aux enchères, y compris sa femme, ce qui lui permet de retrouver sa persona traditionnelle. Et dans Stichus, les épouses ne jouent pas avec leurs maris : elles disparaissent de scène dès l'instant où les adulescentes reviennent. Elles sont d'ailleurs désignées dans les didascalies de la pièce comme sorores, et non comme uxores.

13. Elle est appelée uxor, era, mulier et rarement mater - alors que le senex est surtout désigné comme pater.

14. Quand elle est mariée à un senex. Les épouses de jeune homme n'ont pas d'enfant.

15. Par exemple, Aulularia 535.

16. Par exemple, Miles Gloriosus 690-700.

17. DUmont 1990 : chez Plaute, l'imperium ne désigne pas le pouvoir public et militaire mais le pouvoir domestique, celui d'un père sur son enfant, d'un maître sur son esclave ou d'une femme sur son époux.

18. À l'exception d'Amphitryon, les trois comédies présentant des couples senex-uxor comportent des vieux amoureux. (À ceci près que dans Mercator, l'épouse présente en scène n'est pas celle du senex amator mais celle de son ami. Ce qui entraîne un quiproquo et permet à Plaute d'écrire malgré tout une scène traditionnelle entre une uxor et un senex amator). Ménechme aussi, jeune homme marié à une uxor, est amoureux d'une prostituée. Quant aux deux maris du Stichus, ils ramènent de leur voyage des joueuses de flûtes mais ne se présentent pas comme amoureux. Sur cette comédie qui multiplie les variations, DUPONT et LETESSIER 2012, p. 168-171.

19. Par exemple, Miles Gloriosus $685 \mathrm{sq}$.

20. Par exemple, Asinaria 905 ; Casina 227.

21. Déménète, dans Asinaria aime sa femme quand elle est absente. Quand elle est présente, il la souhaiterait morte (v. 899-900). Dans Aulularia, Mégadore accepte de prendre femme si elle meurt le lendemain du mariage (v. 155-156).

22. Dans Casina, Cleostrata prend clairement le parti de son fils parce qu'elle s'oppose à son mari. Dans Mercator, Dorippa aide le jeune homme malgré elle, de façon très indirecte : en revenant chez elle, elle contrecarre sans le savoir les plans d'un senex. Dans Asinaria, le père et le fils sont associés contre l'épouse qui tient la place de l'opposant. Mais en chassant son mari à la fin de la comédie, elle permet au jeune homme de ne pas avoir à partager les faveurs de la prostituée.

23. Nous entendons par « danse » un mode d'interprétation qui se fait avec une musique jouée à la tibia et qui recourt à l'ensemble du corps. DUPONT et LETESSIER 2012, p. 33-37.

24. L'affrontement entre le senex et son épouse est un proelium (Asinaria, 912) dans lequel le mari craint de recevoir des coups (Asinaria 936 ; Casina 949-950, 970, 1001-1003).

25. Par exemple : Casina 236, 961-962, 967, 977 ; Mercator 705, 709, 748.

26. Sénèque, De ira IV, 1-3.

27. La proximité entre les hommes et les dieux est si grande lors des jeux scéniques qu'on a pu, pour certains jeux nocturnes dédiés aux divinités infernales, empêcher les spectateurs de s'asseoir. SCHEID 2005, p. 100-101.

28. La guerre est déjà un hors scène étonnant mais que l'on retrouve dans d'autres comédies comme Captivi.

29. Jupiter a un bâton (scipio) qui est l'attribut du senex (Amphitruo, 520) et Amphitryon est désigné à deux reprises comme un senex $(1032,1072)$.

30. Ainsi l'apparence seule d'Alcmène devait pouvoir susciter l'amusement du public par son côté inattendu et saugrenu. En revanche, il me paraît difficile de défendre l'idée selon laquelle cette image produirait du comique parce que la femme enceinte est jouée par un homme, comme l'avancent MOORE 1998, p. 120 et CHRISTENSON 2000, p. 38. Tous les rôles de femme étant joués par 
des hommes, l'inhabituel ne provenait que du gros ventre et pas de la conjonction du ventre du rôle et du sexe de l'acteur.

31. Le prologue ne présente donc pas Alcmène comme l'épouse championne de la uirtus aristocratique à laquelle on l'a souvent cantonnée.

32. Par exemple, Pseudolus s'ouvre sur une situation bloquée puisque la fille désirée par le jeune homme a déjà été vendue : ce qui fait la trame traditionnelle d'une intrigue de palliata est donc annulé. Autre exemple, le prologue de Captiui annonce au public que la comédie se fera sans les rôles traditionnels qui font l'action d'une comédie : le proxénète, la prostituée et le soldat.

33. Elle appelle bien son mari mi uir (mon cher époux) mais le public peut d'abord y percevoir une dimension ironique. Voir, par exemple, Casina 974.

34. C'est le cas dans Menaechmi.

35. C'est, par exemple, le cas de la grande scène de ludification qui réunit la fausse Casina et ses deux prétendants : LETESSIER 2011.

36. CHRISTENSON 2000, p. 231. Il cite Properce, 4, 7, 6.

37. Voir les analyses de CHRISTENSON (2001, p. 247-254) sur l'insatisfaction sexuelle d'Alcmène, qui vont dans ce sens, même si l'auteur ne fait pas directement le lien avec la meretrix.

38. Dans Curculio, la pièce commence la nuit: le jeune Phédrome se rend chez le proxénète qui possède la belle esclave dont il est amoureux. Dans Amphitryon (288), la nuit, par sa durée exceptionnelle, est présentée par Mercure comme idéale à passer avec une prostituée.

39. Mercator 501 : Ne plora. Nimis stulte facis; oculos corrumpis talis. Ne pleure pas. C'est trop bête. Abimer de si beaux yeux ! / Amphitryon 530 : Ne corrumpe oculos. N'abime pas tes yeux.

40. Il la désigne comme l'amica, la «copine » du vieux Lysimaque (753) - terme employé par les adulescentes pour désigner leur amoureuse - et comme une compagne de lit experte - scita concubina (758).

41. On retrouve le même refus d'engager la salutation dans Casina. Le vieux Lysidame essaie d'aborder sa femme de façon aimable et celle-ci comprend aussitôt qu'il se joue effectivement d'elle (227-229). Sur la salutation dans le théâtre de Plaute, voir LETESSIER 2000.

42. C'est pourtant la scène qui a été considérée comme la plus proche de la tragédie. Par exemple, MOORE 1998, p. 120.

43. C'est seulement à la toute fin de la tragédie que Jupiter demande à Amphitryon de retrouver ses premiers sentiments pour Alcmène quand il sortira de scène (1141-1142).

\section{RÉSUMÉS}

Partant du constat qu'Alcmène, dans l'Amphitryon de Plaute, est une épouse à part qui ne ressemble pas aux autres personnages féminins des comédies, cet article s'interroge sur ce que recouvre cette "différence ». Plutôt que d'y voir la marque d'une rupture avec la tradition comique, il s'agit de réévaluer cette notion en la réinscrivant au sein du code de la palliata. Ainsi, même si Alcmène n'a pas les caractéristiques conventionnelles d'une épouse sur la scène comique, l'article montre que sa différence ne constitue pas une rupture avec le code mais une façon traditionnelle de s'y inscrire pleinement. Elle constitue, en effet, une variation qui est le mode même de fonctionnement de la codification comique.

Observing that Alcmene, in Plautus'Amphitrio, is a special "wife ", which does not look like the other feminine characters of the Roman comedies, this article focuses on what recovers this 
"difference ». Even if Alcmene has not the conventional characteristics of the wife's role on the comical stage, her difference does not constitute a break with the code, but it appears as a traditional way of joining it completely. It establishes, indeed, a variation, which is the mode of functioning of the comical codification.

INDEX

Mots-clés : Plaute, rôle, palliata, codification, différence, variation, nouveauté

Keywords : Plautus, role, palliata, codification, difference, variation, novelty

\section{AUTEUR}

\section{PIERRE LETESSIER}

Université Paris Diderot-Paris 7, ANHIMA (UMR 8210) 